\title{
General Recommendations and Guidelines for Remote Assessment of Toddlers and Children, in Response to the COVID-19 Pandemic
}

\author{
Kathryn Garrisi $^{1,2 \dagger}$, Carolyn J. King ${ }^{1,2 \dagger}$, Lindsay J. Mullin ${ }^{2 \dagger}$, and Nadine Gaab ${ }^{1,2}$ \\ ${ }^{1}$ Harvard Graduate School of Education, Harvard University \\ ${ }^{2}$ Division of Developmental Medicine, Boston Children's Hospital \\ ${ }^{\dagger}$ These authors contributed equally and are listed alphabetically
}

\section{Author Note}

We have no known conflicts of interest to disclose.

Correspondence concerning this article should be addressed to Nadine Gaab, Harvard Graduate School of Education, Larsen Hall Office 504, 14 Appian Way, Cambridge, MA 02138, United States. Email: nadine_gaab@gse.harvard.edu 


\begin{abstract}
Growing public health concerns and restrictions caused by the COVID-19 pandemic have led to considerable restructuring of children's learning environments and limited access to healthcare services worldwide. The effects of school closures on children's academic achievement remain undetermined; from the perspective of the literature on seasonal learning loss, there is a potential for significant decline in academic performance during this time. Assessment of academic skill and progress is essential for evaluative and diagnostic purposes, especially while restrictions are preventing children from receiving a traditional in-person education. Additionally, clinical and academic evaluations used to inform various services and treatments should not be delayed until families can safely attend in-person visits. A comprehensive protocol for remote developmental and academic assessment of toddlers and school-age children has been established based on the assessment of 180 children, ages 20 months to 9 years, in the United States between May and December, 2020. Software requirements, equipment, setup, administration protocols, general tips, and other considerations regarding virtual evaluations are described. This practical guide for remote assessment can be applied to a variety of settings in educational and clinical fields and has the potential to be especially helpful in identifying learning challenges or providing diagnoses while COVID-19 restrictions persist.
\end{abstract}

Keywords: COVID-19, remote assessment, achievement, developmental assessment, telehealth 


\section{Impact and Implications Statement}

In-person schooling has been interrupted during COVID-19 restrictions, affecting academic progress and counseling services provided in schools. This paper outlines the steps and considerations necessary to remotely administer standardized educational or clinical assessments with toddlers and school-age children. Remote administration will increase access to necessary assessments and progress monitoring, which will assist in addressing academic deficits and clinical needs. 


\section{General Recommendations and Guidelines for Remote Assessment of}

\section{Toddlers and Children, in Response to the COVID-19 Pandemic}

High rates of infection and mortality from the COVID-19 pandemic have led to the sudden closures of educational institutions in more than 190 countries, affecting more than $94 \%$ of students worldwide (United Nations, 2020). Schools, clinics, and research institutions around the world abruptly closed their doors to mitigate a growing public health crisis, postponing critical in-person and research services. As many schools have moved to remote instruction, caregivers have been challenged in adapting to online learning procedures (Dong et al., 2020). While the implications of school closures due to COVID-19 on the academic achievement and progress of students have yet to be determined, COVID-19-related school closures have been proposed to be viewed through the lens of seasonal learning loss usually observed during the summer (Kuhfeld \& Tarasawa, 2020). This seasonal learning loss can have greater and longer lasting effects in lower SES communities (Cooper et al., 1996; Alexander et al., 2007; von Hippel et al., 2018). Remote assessment will provide an opportunity to assess potential learning loss and inform interventions accordingly. Remote assessment will need to be applied to a variety of contexts - such as remote schooling, telemedicine, and teletherapy - as COVID-19 restrictions persist. Such assessment may include screening, diagnostic assessments, and progress monitoring within school and clinical settings.

This paper offers a practical guide to remote assessment for toddler through third grade age groups. Although this protocol was developed for research purposes during the COVID-19 pandemic, it can serve as a template for other research groups, organizations, schools, or clinical entities aiming to conduct assessments virtually for evaluative purposes. This protocol utilizes the same video conferencing platforms that have been adopted worldwide to create online 
curricula (Iivari et al., 2020).

\section{Software for Video-Based Assessment}

A video conference platform is essential for the successful administration of remote assessments or screeners. Zoom Video Communications (https://zoom.us/) was selected to conduct the assessments via video conferencing. The following security features were required in order to comply with institutional requirements: alternative meeting host, meeting password, and virtual waiting room. Although this protocol adopted the Zoom platform, there are other video conferencing software packages available at no cost that are conducive to online assessments. For instance, Google Meet (https://meet.google.com/) has similar features and may be preferred by families given its widespread use in schools. Some institutions or providers may be required to comply with HIPAA regulations (see Dykes, 2020, for details on HIPAA-compliant video conferencing software). Any video recording with these platforms must have prior institutional approval, due to HIPAA and FERPA laws.

In addition to the video conferencing software, use of a commercial remote assessment platform such as Pearson's Q-Global System (Pearson Education, 2021) may be advisable for administration because it provides digital assessment materials and resources for common educational and clinical assessments. However, these platforms can be expensive and out of reach for under-resourced entities. Depending on the copyright of the materials, assessment materials can also be scanned and presented via the screen share feature on a video conferencing platform, eliminating the need to use a paid assessment platform.

\section{Equipment and Setup}

\section{General Setup for the Child}

Since not every family has access to an advanced remote setup, this protocol was 
developed for settings with both ideal and minimal technological options. A checklist of the main setup considerations discussed below can be found in Figure 1.

The most important aspects of the physical setup for the family are a strong Wi-Fi connection or wireless hotspot, minimal noise distractions, and appropriate lighting in the room so that the evaluator can observe the participant clearly during the assessment session. For those with weaker Wi-Fi connections, extra time should be incorporated into the sessions to account for audio or video timeouts, or a Wi-Fi booster should be mailed to the family before the session.

It is important to instruct the family to prepare the child's device before the session in order to optimize productivity and limit distractions. The screen brightness and volume on the child's primary screen should be set at levels that allow the child to clearly hear and see all stimuli. Push notifications should be turned off to avoid distractions and establish a quiet environment. Devices should be plugged in or fully charged prior to the assessment session. The video conferencing window should be maximized to full screen on the child's computer. The caregiver should also minimize "self-view" so that the child is not distracted by his or her own image. Finally, the caregiver should make sure that the view of the evaluator on the child's screen is not superimposed on top of the shared screen view, obscuring the stimuli presented.

The use of two screens with video capabilities for remote video conferencing has been shown to be an ideal setup. The primary screen, often a computer, should be used to display stimuli to the child and show the evaluator's face. The secondary screen, usually a smaller device such as a smartphone or tablet, should be placed to the side of the child and connected to the video conferencing platform over video only, to show the evaluator where the child is pointing to on the primary screen. Please see Figure 2 for an example of the advanced assessment setup.

However, this setup is not always a possibility. Some families prefer to use a second 
computer rather than a smartphone or tablet as their second device, which does not interfere with administration as long as the table used can accommodate them both. Alternatively, some families have only two small devices (e.g., two tablets, or a tablet and a phone) to use during the session. This is viable as long as the child can clearly see the stimuli presented on the primary screen, which should be confirmed prior to the session. For families whose devices are used by multiple people, it is important to accommodate everyone's various commitments when scheduling assessment sessions. To prevent scheduling conflicts, it may be helpful to have those families use school-issued devices (e.g., Chromebooks) whenever possible. For homes with only one device available, the annotation tools included in some video conferencing platforms, more active involvement of the caregiver, and adaptation by the evaluator has proven helpful. Because children as young as Kindergarten age have been shown to operate a computer mouse reliably (Donker \& Reitsma, 2007), the annotating feature of some video conferencing platforms allows a child to mark the part of the screen he or she would otherwise point to. Evaluators should then clear the annotated marks after the response is recorded. Please see Figure 3 for further explanation of the annotation tool. In other circumstances, the caregiver should act as a proxy for the child, indicating the child's response to the evaluator when he or she is required to point to the primary screen. For example, if there are three items on the screen labeled by numbers, the caregiver can indicate the number associated with the response that the child chose.

Alternatively, the evaluator can also allow the child to indicate which item or item number (for older children who know symbolic numbers) he or she chose.

\section{Setup for Toddler Sessions}

Most assessment items in developmental assessments for toddlers can be classified into two categories: observation or evoked response. To best support caregiver facilitation of 
standardized assessments, the order in which items are administered could be modified if the assessment protocols allow this. For instance, it is advisable to start with a "natural play" period, in which caregivers are asked to play as they typically would with their toddler on a carpet or play mat, and simply allow the evaluators to observe through the video conferencing platform.

Prior to the session, the evaluator and caregiver should identify the best place to put the device so that the camera captures the entire play area. Even though the environment and methodologies are adjusted during virtual assessments, child language samples are comparable to in-person assessment sessions (Manning et al., 2020). During this play period, some language and motor skills can be observed.

A second period, "structured play" at a table or desk (setup is described in the previous section) can follow the initial play period during which the caregiver can be prompted to repeat questions or instructions verbatim to his or her toddler, in order to collect items that require an evoked response. Separating the session into these two parts allows for ease of caregiver facilitation and reduced overall assessment time.

To replicate assessment materials, caregivers can be asked to provide typical household objects (e.g., blocks, ball, board book) that resemble those included in the standardized assessment kit. Additionally, if objects are not available in the household, pictures of the objects can be compiled into a slideshow presentation and presented using the screen share feature of the video conferencing platform.

\section{Equipment and Setup for the Evaluator}

The evaluator may use two devices or one device with supplemental materials for administration. If the evaluator chooses to use two devices, the stimuli should be displayed on the first device and shared with the child via the screen share feature using the chosen video 
conferencing platform. The evaluator prompts (pulled from the administration platform or scanned from the physical administration manuals) should be displayed on the second device for the evaluator to read and should not be connected to the video conferencing session. Using this setup, the evaluator would not need a physical copy of the manuals.

The evaluator may also opt to use one computer screen and hard copies of evaluator manuals. The stimuli should be displayed via the screen share feature of the video conferencing platform. The evaluator should read the evaluator prompts directly from the physical copies of the administration manuals. Both of these evaluator setup options have proven successful.

\section{Protocol}

\section{Sessions with Toddlers}

It is advisable to separate a toddler session into an observation-based, "natural play" period, followed by a "structured play" period for items that require an evoked response. An introductory call with the caregiver before the assessment session is especially critical for the remote assessment of toddlers, particularly if the caregiver is asked to facilitate parts of the assessment session.

Many developmental assessments that are standardized for infant and toddler ages have items that can be scored based on caregiver report. However, during virtual sessions, caregivers are juggling behavior management and session facilitation. Although it would still be optimal to try to elicit those responses during the assessment session, it can be helpful to have a list of questions ready that are able to be scored by caregiver report in the introductory call. If the evaluator already has the caregiver's confirmation that the child reliably performs a certain action, this could streamline the assessment session by eliminating interruptions of questions throughout. 
During the "structured play" portion of a typical in-person assessment session, many items that utilize a pictorial stimuli book require the evaluator to point to the stimuli while asking a question about it. For example, if one image contains an apple and a ball, and the evaluator is asking the child to name the apple, he or she would point to the apple and say, "What do you call this?" To replicate this action in a virtual setup, the evaluator can inform the caregiver that when asking about a specific item, such as the apple, the evaluator will circle it on the screen with their cursor. Given that toddlers have a "video deficit" and learn more reliably from in-person gestures than videos (Anderson \& Pempek, 2005), caregivers should be asked to point to the item on the screen that the evaluator is circling while repeating the question verbatim.

\section{Sessions with School-Age Children}

It is important to instruct the caregiver to avoid interfering with the administration of, or response to, any assessment items. It should be emphasized that his or her role is to assist with technology, manage behavior, and request breaks if applicable - while refraining from providing answers, feedback, or excessive praise.

For assessments that include auditory stimuli, pre-recorded stimuli can be added to a slideshow presentation. For assessments that require writing, children can be asked to write on a blank sheet of paper and show it to the camera so that the evaluator can record the answers. An alternative approach is to provide answer sheets through the mail or email and ask the caregiver to print them out prior to the session. However, the evaluator must emphasize that children should not make themselves familiar with these assessment sheets or stimuli prior to the assessment session, as this can interfere with the standardized assessment protocols.

\section{Behavioral Management}

Successful assessment sessions often rely on assistance from a caregiver. The evaluator 
cannot control a virtual assessment environment in the same way that he or she could if it were conducted in-person. As a result, the evaluator requires the caregiver to help control the physical assessment environment and help motivate the child.

In the introductory call with the caregiver, it is important to set behavioral expectations for the session and enlist assistance in managing the child's compliance and attention. For sessions with toddlers, caregivers can be asked to assist with behavioral challenges and session facilitation. For sessions with school-age children, caregivers should eliminate distractions from the environment, redirect their child's attention, or suggest short breaks as necessary. Some children benefit from a one-on-one setup with the evaluator to avoid distractions by the caregiver or to ensure that the caregiver or sibling is not helping to answer questions or exerting pressure on the child. Some children are motivated by rewards as appropriate, which can be given with the coordination of the evaluator and the caregiver.

\section{Challenges of Remote Assessment}

Behavioral management tends to be more difficult to address during remote assessment sessions, as the evaluator is physically separate from the child. Providing movement breaks and progress tracking while video conferencing can feel disingenuous or less motivating for the child. Children may accidentally tamper with the setup of their devices and become distracted. Maintaining a quiet environment can be challenging, especially when other siblings in the home may distract or provide unsolicited answers or advice. It is also important to be aware of an increased number of behavioral challenges in remote environments due to the unfamiliarity of the remote assessment situation or the lack of time to build proper rapport. This protocol has been successfully administered with just one screen on a desk to accommodate space constraints in some households. 
Technological issues or failures create additional interruptions to the assessment session. Wi-Fi cutouts and audio delays can be unavoidable; a protocol should be established prior to the assessment session that outlines specific actions to be taken if technological challenges occur. For instance, many assessments contain specific instructions regarding when and how to repeat prompts. However, technological challenges may lead to "frozen" screens or involuntary interruptions that may warrant repeating prompts.

The presence of caregivers or older siblings throughout the assessment sessions can be instrumental but does provide unique challenges during the assessment sessions. Finding the right balance between having the caregiver as a silent observer and an essential assessment facilitator can be challenging at times.

Most of the aforementioned challenges of remote assessment can be easily controlled for to ensure validity and reliability of the assessment session. However, it is important to emphasize that the evaluator needs to determine whether the child's remote assessment session reflects his or her true skill level and whether the assessment can be classified as valid and reliable. In instances where the validity of the data becomes compromised, the evaluator may end the session early and consider a second session. Patience and understanding of these challenges are paramount to the successful completion of assessments needed to ensure validity and reliability. Available assessment platforms may have specific guidelines that can assist in determining whether the assessment is valid for making a diagnosis or determining risk statuses.

\section{Tips for Successful Online Assessment}

- Have the caregiver test his or her setup during the introductory call to minimize delays or technological difficulties during the session.

- Minimize and control break times to prevent children from losing focus (as recommended 
with in-person sessions).

- Remind caregivers of expectations at the beginning of the session: redirect the child as necessary, avoid giving unsolicited prompts or answers, and minimize distractions in the room.

- Get creative with children that require more motivation to stay focused through the assessments. Successful motivation tools include: a virtual checklist or sticker chart, showing the child a new toy and taking a minute to chat about the toy, or simply letting the child know how many more games they have left may help to remind them that the session is ending.

- If possible, avoid scheduling a session directly after a child has had a full day of online school. As a result of COVID-19, children have had significantly more screen time per day than before the start of the pandemic (Lau \& Lee, 2020). Children can often get fatigued with back-to-back video calls and ultimately may not perform at their true skill level.

- The evaluator should be fluent in the language that the caregiver is most comfortable with, to ensure that the caregiver can fully understand the expectations and procedures.

- Depending on how many assessments will be administered, the evaluator should plan for more than one assessment session. This can relieve significant time pressure for the evaluator, child, and caregiver. If the first session is not running smoothly, the session can be ended early and outstanding assessments can be moved to another session. Many children perform and behave better in multiple, short sessions.

\section{Additional Considerations Specific to COVID-19}

Families may be experiencing undue stress from the COVID-19 pandemic. The evaluator 
should consider trauma, loss of a family member, and adverse financial changes, as possible confounding influences during this process.

The literacy environment and practices in the home may influence learning loss while COVID-19 restrictions are in place and should be considered in the evaluation of a child's performance on assessments. A questionnaire that can assess these effects has been developed (see King et al., 2020, for details).

The extent of learning loss due to school closures from COVID-19 restrictions must be taken into account. Some children may experience full-time schooling, a hybrid learning environment, or fully remote schooling. Additionally, remote instruction can vary in quality and quantity and, as a result, should be considered when interpreting the results of the assessment.

\section{Discussion}

Remote assessments offer the opportunity to assess academic progress and overall development in a remote or hybrid learning environment - such as those resulting from COVID19 restrictions and school closures. Here, practical tips and recommendations are provided for the remote assessment of toddlers through elementary-age children that can be adapted for various educational and clinical settings.

Learning loss due to COVID-19 restrictions has the potential to be detrimental to children's academic achievement. Measuring the extent of these deficits through standardized assessments could inform accommodations and interventions and mitigate the long-term effects of lost education. Those providing psychological services in schools could implement this remote protocol for assessments typically conducted in-person. Remote assessment provides a setting for schools to continue to evaluate children's development safely, and in a standardized manner. 


\begin{abstract}
About Us
The Gaab Lab is a developmental cognitive neuroscience research lab located at the Harvard Graduate School of Education (located at Boston Children's Hospital prior to 1/1/21). The lab conducts research involving behavioral assessment and screening of multiple domains of development and functioning in children with and without risk for developmental dyslexia, from infancy through school age. Due to the COVID-19 pandemic, it was no longer possible to conduct in-person research assessments. As a result, protocols were developed at Boston Children's Hospital that enabled the continued administration of assessments remotely. These protocols were successfully administered to 180 children of different ages, races, and socioeconomic backgrounds located throughout the United States between May and December of 2020, and these suggestions can effectively aid in the developmental assessment of children in various settings.
\end{abstract}




\section{Figure 1}

Checklists for Remote Assessment Setup

\section{General Setup Checklist}

\section{Introductory Call}

$\square$ Explain the goals and setup of the session

$\square$ Help determine what setup will work best for this family (including a minimally distracting room with a table or desk, Wi-Fi connectivity etc. listed below, and 1-2 devices that can connect to the video conferencing platform)

\section{Setup of the Family's Physical Space}

$\square$ Strong Wi-Fi connection or wireless hotspot

$\square$ Setup at a table or desk

$\square$ Minimal noise distractions

Appropriate lighting

Setup of the Family's Primary Computer Screen

$\square$ Screen brightness is turned up

$\square$ Volume is appropriately set

$\square$ Notifications on device(s) are disabled

$\square$ Device(s) are plugged in or fully charged

$\square$ Video conferencing window is maximized to full screen

$\square$ If possible, the "self-view" is minimized (so that the child is not distracted by his or her own image)

$\square$ View of the evaluator on the child's screen is not superimposed on top of the shared screen view (can often be achieved by entering into full-screen view and then hitting the "Escape" button on the keyboard)

\section{Setup if the Child Needs to Point to Stimuli}

- If the family has a second device available (See Figure 2), the device is

$\square$ Placed to the side of the child

$\square$ Connected to the video conferencing platform over video only

- If the family does not have a second device available

$\square$ Be prepared to either use the annotating feature of the video conferencing platform (See Figure 3), or ask for the caregiver or child themself to verbalize which part of the screen they would have otherwise pointed to

\section{Caregiver Involvement}

$\square$ Not to interfere or respond for the child

$\square$ Assist with technology

$\square$ Manage behavior 
$\square$ Request breaks

\section{Toddler Setup Checklist}

Plan to separate the session into two parts: an observational "natural play" period, and a "structured play" period for items requiring an evoked response

\section{For "Natural Play"}

$\square$ Setup at a typical play area (carpet or play mat) and toys

$\square$ Make sure the camera connected to the video conferencing platform has a view of the entire play area

$\square$ Instruct the caregiver to play with their toddler as they typically would

\section{For "Structured Play"}

$\square$ Setup at a table or desk as described in the "General Setup" above

Instruct the caregiver to repeat the evaluator's questions or instructions verbatim

Note. These checklists are the key points for overarching setup considerations. The paper's recommendations should be reviewed for additional information. 
Figure 2

Advanced Remote Setup.
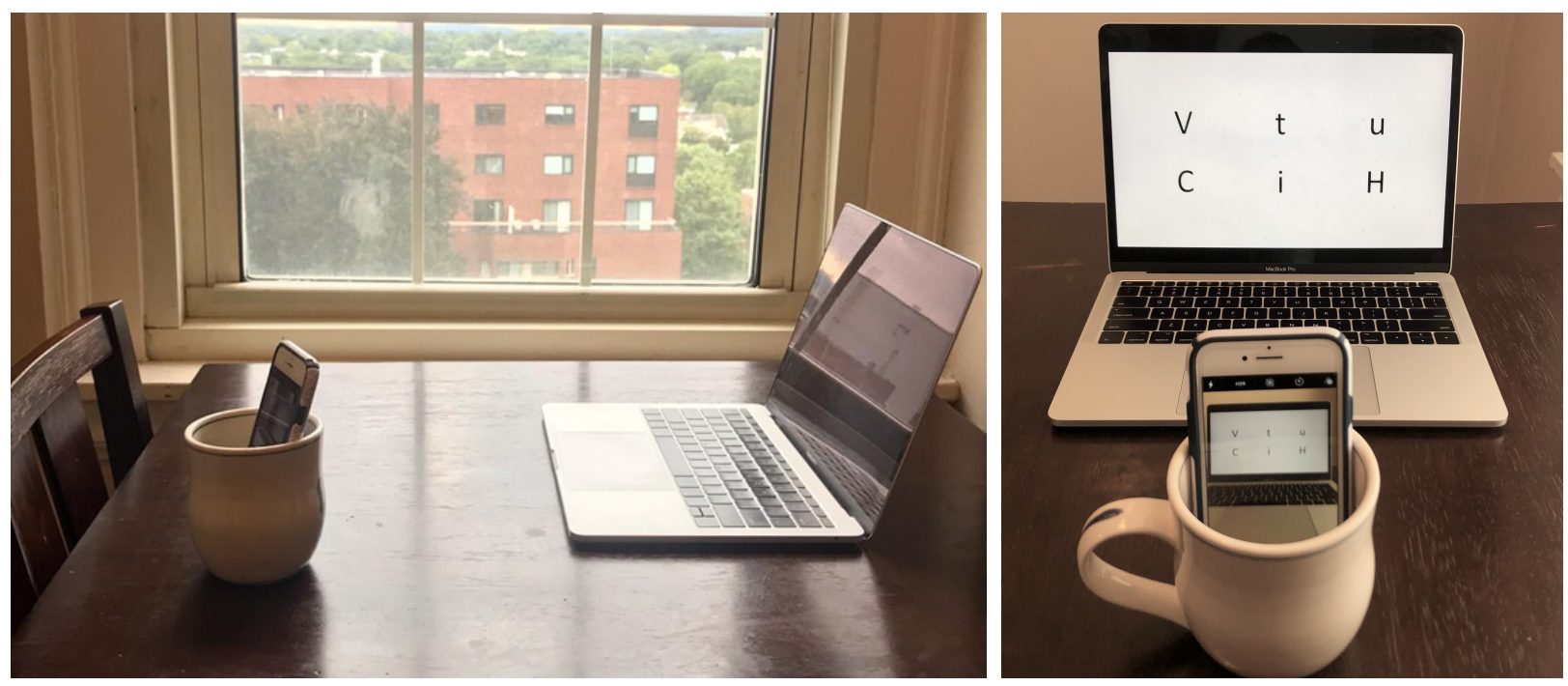

Note. The ideal setup of a virtual assessment session for school-aged children and the "structured play" portion of a toddler session utilizes two devices. Both devices should be connected to an online video conferencing platform, enabling the evaluator to see both the child's face (via the laptop's camera) and where the child points (via the smartphone's camera). The child should view the stimuli screen shared by the evaluator on the primary device (laptop here). 


\section{Figure 3}

Example Use of the Annotation Tool.

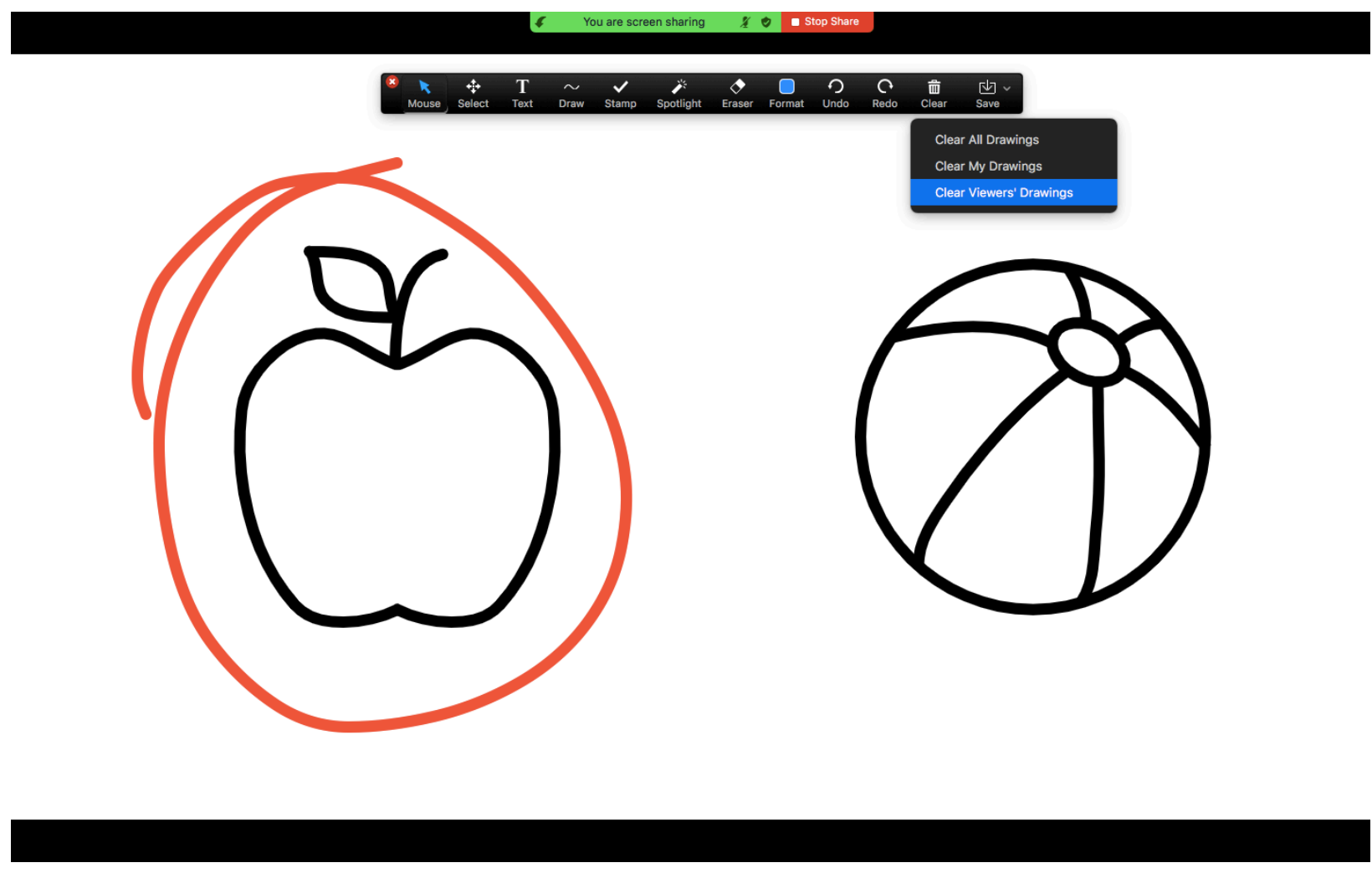

Note. The annotation tool of some video conferencing platforms can indicate a child's response to an item they would otherwise point to during an in-person session. Here, the evaluator asked, "Which of these is an apple? Circle the apple." The child's response is shown from the evaluator's perspective, as the hand-drawn circle around the apple on the left. Once the response is recorded, the evaluator should erase the annotation, using the highlighted button that reads

“Clear Viewer Drawings” on this platform. 


\section{References}

Alexander, K. L., Entwisle, D. R., \& Olson, L. S. (2007). Lasting consequences of the summer learning gap. American Sociological Review, 72 (2), 167-180.

Anderson, D. R., \& Pempek, T. A. (2005). Television and very young children. American Behavioral Scientist, 48, 505-522.

Cooper, H., Nye, B., Charlton, K., Lindsay, J., \& Greathouse, S. (1996). The effects of summer vacation on achievement test scores: A narrative and meta-analytic review. Review of Educational Research, 66 (3), 227-268.

Dong, C., Cao, S., \& Li, H. (2020). Young children's online learning during COVID-19 pandemic: Chinese parents' beliefs and attitudes. Children and Youth Services Review, $118,105440$.

Donker, A., \& Reitsma, P. (2007). Young children's ability to use a computer mouse. Computers and Education, 48(4), 602-617.

Dykes, A. (2020, April 10). The Best HIPAA Compliant Video Conferencing Tools for Telehealth. Retrieved January 14, 2021, from https:/technologyadvice.com/blog/healthcare/hipaa-video-conferencing-tools/

Iivari, N., Sharma, S., \& Ventä-Olkkonen, L. (2020). Digital transformation of everyday lifeHow COVID-19 pandemic transformed the basic education of the young generation and why information management research should care?. International Journal of Information Management, 55, 102183.

King, C. J., Lee, A., Zuk, J., Ravi, N., \& Gaab, N. (2020, September 3). The COVID-19 Home Environment Literacy Practices (COVID19-HELP) Questionnaire. https://doi.org/10.31219/osf.io/2bjhd 
Kuhfeld, M. \& Tarasawa, B. (2020). The COVID-19 slide: What summer learning loss can tell us about the potential impact of school closures on student academic achievement [Brief]. NWEA. https://www.nwea.org/content/uploads/2020/05/Collaborative-Brief_Covid19-Slide-APR20.pdf

Lau, E. Y. H., \& Lee, K. (2020). Parents' Views on Young Children's Distance Learning and Screen Time During COVID-19 Class Suspension in Hong Kong. Early Education and Development, 118.

Manning, B. L., Harpole, A., Harriott, E. M., Postolowicz, K., \& Norton, E. S. (2020). Taking Language Samples Home: Feasibility, Reliability, and Validity of Child Language Samples Conducted Remotely With Video Chat Versus In-Person. Journal of speech, language, and hearing research: JSLHR, 63(12), 3982-3990.

https://doi.org/10.1044/2020_JSLHR-20-00202

Pearson Education. (2021). Q-Global System. NCS Pearson, Inc.

https://www.pearsonassessments.com/professional-assessments/digital-solutions/qglobal/about.html

United Nations (2020). Policy Brief: Education during COVID-19 and beyond [Policy brief]. United Nations. https://www.un.org/development/desa/dspd/wpcontent/uploads/sites/22/2020/08/sg_policy_brief_covid-19_and_education_august_2020.pdf von Hippel, P. T., Workman, J., \& Downey, D. B. (2018). Inequality in reading and math skills forms mainly before kindergarten: A replication, and partial correction, of "Are schools the great equalizer?" Sociology of Education, 91(4), 323-357. 